\title{
Characteristics, Comorbidities, and Outcomes of SARS-CoV-2 Infection in Patients With Autoimmune Conditions Treated With Systemic Therapies: A Population-based Study
}

\author{
Jeffrey R. Curtis ${ }^{1}(\mathbb{D})$, Xiaofeng Zhou²(D), David T. Rubin ${ }^{3}$ (D), Walter Reinisch ${ }^{4}$ (D), Jinoos Yazdany ${ }^{5}$ (D),

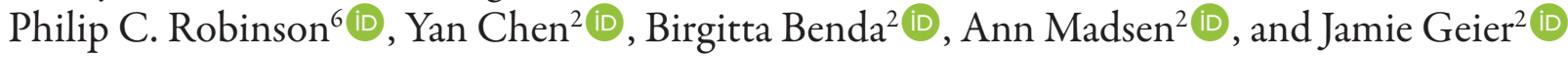

ABSTRACT. Objective. To describe characteristics and coronavirus disease 2019 (COVID-19) clinical outcomes of patients with rheumatoid arthritis (RA), psoriatic arthritis (PsA), or ulcerative colitis (UC) receiving systemic therapies vs the general population.

Methods. This descriptive retrospective cohort study used data from the United States Optum deidentified COVID-19 electronic health record dataset (2007-2020). Adults with COVID-19 were stratified into 3 disease cohorts (patients with RA, PsA, or UC who had received systemic therapy) and a comparator cohort not meeting these criteria. Incidence proportions of hospitalization and clinical manifestations of interest were calculated. Using logistic regression analyses, risk of endpoints was estimated, adjusting for demographics and demographics plus comorbidities.

Results. This analysis (February 1 to December 9, 2020) included 315,101 patients with COVID-19. Adjusting for demographics, COVID-19 patients with RA $(\mathrm{n}=2306)$ had an increased risk of hospitalization (OR 1.54, 95\% CI 1.39-1.70) and in-hospital death (OR 1.61, 95\% CI 1.30-2.00) compared with the comparator cohort $(n=311,563)$. The increased risk was also observed when adjusted for demographics plus comorbidities (hospitalization OR 1.25, 95\% CI 1.13-1.39 and in-hospital death OR 1.35, 95\% CI 1.09-1.68]). The risk of hospitalization was lower in COVID-19 patients with RA receiving tumor necrosis factor inhibitors (TNFi) vs non-TNFi biologics (OR 0.32, 95\% CI 0.20-0.53) and the comparator cohort (OR $0.77,95 \%$ CI $0.51-1.17$ ). The risk of hospitalization due to COVID-19 was similar between patients receiving tofacitinib and the comparator cohort.

Conclusion. Compared with the comparator cohort, patients with RA were at a higher risk of more severe or critical COVID-19 and, except for non-TNFi biologics, systemic therapies did not further increase the risk. (ENCePP; registration no. EU PAS 35384)

Key Indexing Terms: COVID-19, psoriatic arthritis, rheumatoid arthritis, ulcerative colitis

This study was sponsored by Pfizer Inc.

${ }^{1} J$.R. Curtis, MD, Division of Clinical Immunology and Rheumatology, University of Alabama at Birmingham, Birmingham, Alabama, USA; ${ }^{2} X$. Zhou, PhD, Y. Chen, MD, B. Benda, MD, PhD, A. Madsen, PhD, J. Geier, PhD, Pfizer Inc, New York, New York, USA; ${ }^{3} D . T$. Rubin, MD, University of Chicago Medicine Inflammatory Bowel Disease Center, Chicago, Illinois, USA $;{ }^{4}$ W. Reinisch, MD, Medical University of Vienna, Vienna, Austria; 5 J. Yazdany, MD, Division of Rheumatology, Department of Medicine, University of California, San Francisco, California, USA; ${ }^{6}$ P.C. Robinson, MBChB, PhD, School of Clinical Medicine, Faculty of Medicine, The University of Queensland, Herston, Queensland, Australia.

$J R C$ has received grant support from, and has been a consultant for, Pfizer Inc. DTR has received grant support from Takeda; and has received consulting fees from AbbVie, AbGenomics, Allergan Inc, Boebringer Ingelheim, BMS, Celgene Corp/Syneos, Check-Cap, Dizal Pharmaceuticals, Eli Lilly, GalenPharma/Atlantica, Genentech/Roche, Gilead Sciences, GSK, Ichnos Sciences S.A., Janssen, Narrow River Management, Pfizer Inc, Prometheus Laboratories, Reistone, Shire, Takeda, and Techlab Inc. WR has received grant support from Abbott, AbbVie, AESCA, Centocor, Dr. Falk Pharma, Immundiagnostik, and MSD; has received lecture fees from Abbott, AbbVie, AESCA, Aptalis, Celltrion, Centocor, Danone, Dr. Falk Pharma, Elan, Ferring Pharmaceuticals, Immundiagnostik, Mitsubishi Tanabe Pharma, MSD, Otsuka, PDL, Pharmacosmos, Schering-Plough, Shire, Takeda, Therakos, Vifor, and Yakult; and has received consulting fees from Abbott,
AbbVie, AESCA, Amgen, AM Pharma, Astellas, AstraZeneca, Avaxia Biologics, Bioclinica, Biogen Idec, Boehringer Ingelheim, BMS, Celgene, Cellerix, Celltrion, Centocor, ChemoCentryx, Covance, Danone, Dr. Falk Pharma, Elan, Ferring Pharmaceuticals, Galapagos, Genentech, Gilead Sciences, Grünenthal, ICON, Index Pharma, Inova, Janssen, Johnson \& Johnson, Kyowa Hakko Kirin Pharma, Lipid Therapeutics, MedImmune, Millennium, Mitsubishi Tanabe Pharma, MSD, Nestlé, Novartis, Ocera, Otsuka, PDL, Pfizer Inc, Pharmacosmos, Procter \& Gamble, Prometheus Laboratories, Robarts Clinical Trials, Schering-Plough, Second Genome, SetPoint Medical, Takeda, Therakos, TiGenix, UCB, Vifor, Zyngenia, and $4 S C$. JY has received consulting fees from AstraZeneca and Eli Lilly; and has received a research grant from AstraZeneca. PCR has received personal fees from AbbVie, Atom Biosciences, Eli Lilly, Gilead Sciences, Janssen, Novartis, Pfizer Inc, Roche, and UCB; and has received grant support from Janssen, Novartis, Pfizer Inc, and UCB. XZ, YC, BB, and AM are employees and shareholders of Pfizer Inc. JG was formerly employed by Pfizer Inc.

$J R C, X Z, B B, A M$, and JG contributed to the conception and design of the study. All authors contributed to the acquisition, analysis and interpretation of the data, critically revised each draft of the manuscript for intellectual content, read and gave approval of the final version submitted for publication and accept accountability for the accuracy and integrity of the work.

Address correspondence to Dr. X. Zhou, Pfizer Inc, 235 East 42nd Street, New York, NY 10017, USA. Email:xiaofeng.zhou@pfizer.com.

Accepted for publication October 26, 2021. 
Patients with rheumatoid arthritis (RA), psoriatic arthritis (PsA), and ulcerative colitis (UC) usually require treatment with immunomodulatory medications, which may increase the risk of SARS-CoV-2 and other infections. ${ }^{1,2,3}$ Moreover, these patients may be at higher risk for more severe coronavirus disease 2019 (COVID-19), including hospitalization, complications, and death. The American College of Rheumatology (ACR) asserts that patients with autoimmune and inflammatory diseases treated with immunomodulatory therapies seem to be at higher risk for more severe COVID-19, but the lack of evidence from large, population-based studies is noted. ${ }^{4}$ The effect of immunomodulation on the severity of COVID-19 remains unclear, and whether interruption of immunomodulatory therapies prevents severe complications of COVID-19 lacks high-quality evidence.

This study is a voluntary postauthorization safety study for tofacitinib embedded within the Optum deidentified COVID-19 electronic health record (EHR) dataset (2007-2020), for which data are derived from healthcare provider organizations in the United States. ${ }^{5}$ Utilizing data from the Optum COVID-19 EHR dataset, the objective of this population-based study was to assess whether systemic therapies, including tofacitinib, modify the risk of serious clinical manifestations and outcomes of COVID-19. Tofacitinib is a Janus kinase (JAK) inhibitor approved for the treatment of RA, PsA, and UC at the time of study start.

We report data on baseline characteristics, comorbidities, and clinical outcomes from COVID-19 patients with RA, PsA, or UC treated with systemic therapies and a comparator cohort representative of the general population who also had COVID-19.

\section{METHODS}

Study design. This was a descriptive, retrospective cohort study of patients with RA, PsA, and UC, utilizing data from the Optum COVID-19 EHR (from February 1 to December 9, 2020) and is a voluntary postauthorization safety study of tofacitinib conducted by Pfizer Inc. The Optum COVID-19 EHR dataset was curated specifically to provide access to near real-time, structured, longitudinal health data for patients tested for or diagnosed with SARS-CoV-2 infection (Supplementary Methods, available with the online version of this article) and is a subset of Optum EHR data, which reflects the general demographic distributions (e.g., age and gender) of the US population. This dataset includes deidentified claims and clinical data from multiple health plans and healthcare providers (specialist and primary care physicians), and includes plan enrollment information, medical and pharmacy claims (inpatient and outpatient), and laboratory results from multiple payers integrated across care settings and longitudinally linked at the patient level. ${ }^{5}$ All patients within the Optum COVID-19 EHR dataset had exposure to or had been tested for SARS-CoV-2, or had a COVID-19 diagnosis. Further, this longitudinal dataset included data from 2007, and thus provided a patient medical history for analysis.

This study is a postauthorization safety study using data from a secondary database study of deidentified structured data and, hence, independent ethics committee and/or institutional review board review was not required.

Patient analysis. Patients were identified using the following selection criteria: age $\geq 18$ years at index date (date of COVID-19 diagnosis), $\geq 6$ months of continuous database enrollment before index date (ie, $\geq 1$ healthcare activity within 6 months before index date), and a diagnosis of COVID-19 (defined as a diagnosis code for SARS-CoV-2, a positive test for SARS-CoV-2 active infection [antigen and/or PCR], and/or a positive antibody test). Patients meeting the selection criteria were subdivided into 3 tofacitinib-indicated cohorts and 1 comparator cohort. The indicated cohorts included patients who had a clinical diagnosis of RA, PsA, or UC (using the International Classification of Diseases, 10th revision, Clinical Modification [ICD-10-CM] codes) within 2 years before index date and had evidence of treatment with conventional synthetic disease-modifying antirheumatic drugs (csDMARDs), JAK inhibitors (including tofacitinib), tumor necrosis factor inhibitors (TNFi), or non-TNFi biologics, prescribed within 2 years before index date (Supplementary Methods, available with the online version of this article), but may have discontinued treatment before COVID-19 diagnosis. Indicated cohort patients were further subdivided based on baseline ( 6 months before index date) systemic therapy. Treatment groups were mutually exclusive and patients who switched therapy during the baseline period were excluded. Disease cohorts were mutually exclusive and patients with an overlapping diagnosis of RA, PsA, or UC within 2 years before index date were excluded.

The general population comparator cohort included patients with COVID-19 who had no evidence of a diagnosis of RA, PsA, or UC, or use of systemic therapies (those listed for indicator cohort eligibility), within 2 years before index date.

Study outcomes. Baseline (within 6 months before index date) patient demographics, comorbidities, and systemic therapies were evaluated. The primary endpoints of this study were hospitalization and intensive care unit (ICU) admission within 30 days of COVID-19 diagnosis. Clinical manifestations or outcomes of interest within 30 days of COVID-19 diagnosis included in-hospital death, pneumonia, kidney failure, thrombotic events, acute respiratory distress syndrome (ARDS), heart failure, and sepsis or septic shock (Supplementary Methods, available with the online version of this article). Other clinical manifestations or outcomes of interest included all-cause mortality (within 90 days of COVID-19 diagnosis), mechanical ventilation or extracorporeal membrane oxygenation (ECMO; among patients hospitalized within 30 days of COVID-19 diagnosis), and intravenous Ig (a proxy for severe COVID-19 at the time of study initiation).

Statistical methods. The main summary measures of this study included means (with SDs) and medians for continuous variables. Numbers and percentages were calculated for dichotomous or categorical variables. The proportions of patients (incidence proportions with 95\% CIs) with primary endpoints and outcomes of interest within the indicated and comparator cohorts were calculated. Incidence of ICU admission and in-hospital death was further conditional on hospitalization. Indicated cohorts were stratified by immunological condition and baseline treatment (TNFi, non-TNFi biologics, tofacitinib, JAK inhibitors, and csDMARDs). No imputation for missing values was performed.

In an exploratory analysis, the risk of primary endpoints and outcomes of interest between the indicated and comparator cohorts was estimated using logistic regression models. The first model was adjusted for demographics (age, sex, race, and region) only and the second model for demographics plus comorbidities (lung disease [interstitial lung disease (ILD), asthma, and chronic obstructive pulmonary disease], venous thromboembolism, hypertension, coronary artery disease, serious infections [hospitalized], cancer, diabetes, chronic kidney disease [CKD] or dialysis, and liver disease). Selection of the covariates in a second model was based on clinical relevance, data availability, and whether a comorbidity should be characterized as a mediator (ie, an effect of the indicated condition that might result in more severe COVID-19) or a confounder (ie, a variable that is associated both with the risk of the indicated condition and the risk of COVID-19). Given the sparsity of the data, logistic regression models with adjustment for demographics plus comorbidities were restricted to those endpoints with $>30$ events for each endpoint or outcome in the RA, PsA, and UC cohorts. The risk of hospitalization (using logistic regression models adjusting for age, sex, race, region, hypertension, diabetes, and CKD 
or dialysis) was further estimated in the RA cohort, comparing patients receiving each baseline systemic immunomodulatory therapy and with the comparator cohort. The same analyses were performed with patients from the 3 indicated cohorts combined. ORs and $95 \%$ CIs were reported for each of the outcomes.

\section{RESULTS}

Patients and baseline characteristics. The Optum COVID-19 EHR dataset included 3,299,778 patients (as of December 9, 2020), of whom 315,101 (9.5\%) met the inclusion and exclusion criteria (Figure 1).

Of patients meeting the inclusion criteria, 164,276 (52.1\%) had a diagnosis code for SARS-CoV-2 and a positive test for SARS-CoV-2 active infection, 77,182 (24.5\%) had a positive test for SARS-CoV-2 active infection, and 73,643 (23.4\%) had a diagnosis code for SARS-CoV-2.

Baseline characteristics for each cohort are presented in Table 1 and in Supplementary Table 1 (available with the online version of this article). Patients with COVID-19 in the indicated cohorts were generally older and had more comorbid conditions than those in the comparator cohort, and the RA cohort had a higher proportion of females and patients with Black or African American ethnicity.

In patients with RA aged $\geq 76$ years, the highest proportion of patients received non-TNFi biologic prescriptions compared with other medications, and those with a prescription for non-TNFi biologics also had the highest proportion of baseline comorbidities, compared with patients receiving tofacitinib, TNFi, or csDMARDs. A similar trend was noted in the PsA and UC cohorts (Supplementary Table 1, available with the online version of this article).

Diagnoses were more likely to be provided within the emergency room setting, compared with inpatient or outpatient settings (Supplementary Figure 1, available with the online version of this article).

Crude incidence of hospitalization and ICU admission. The crude incidence of hospitalization due to COVID-19 and ICU

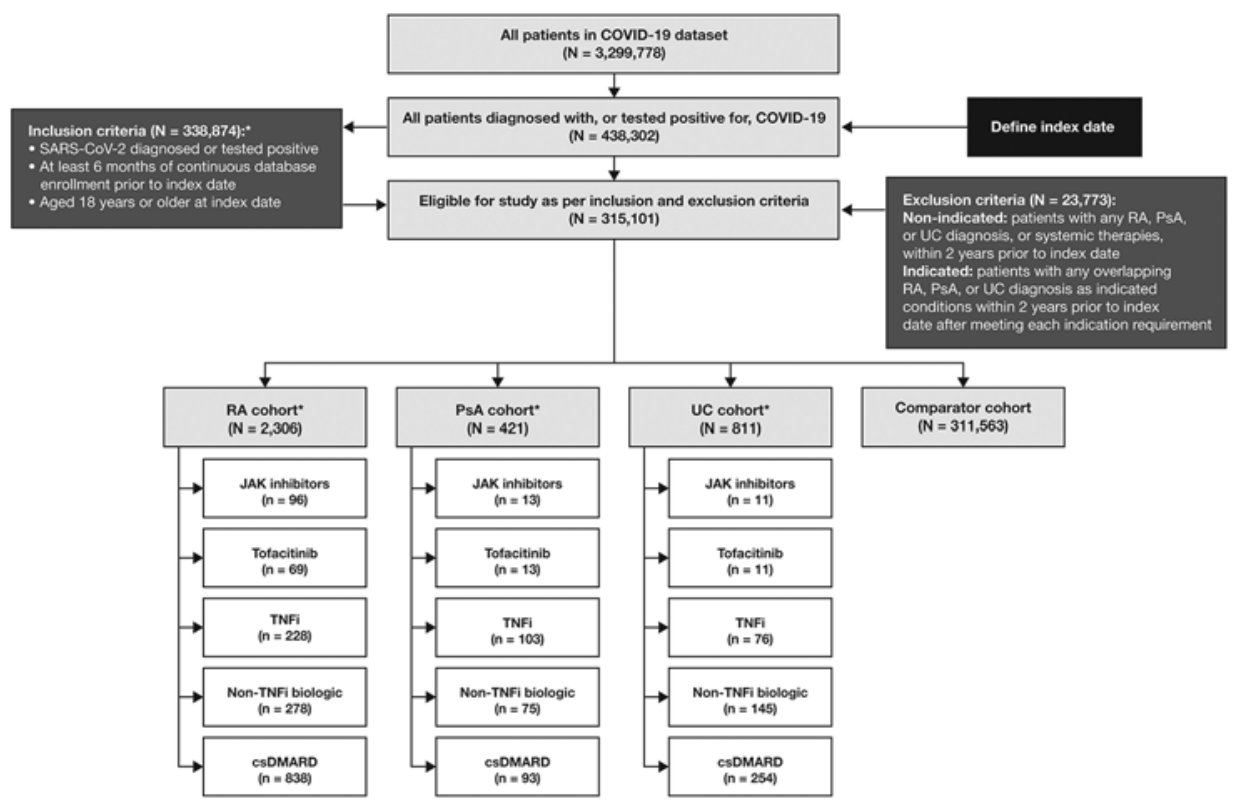

Figure 1. Selection of patients with COVID-19 for the indicated and comparator cohorts. " Indicated cohort eligibility was based on receipt of clinical diagnosis of RA, PsA, or UC (using International Classification of Diseases, 10th revision, Clinical Modification codes) and systemic therapy within the 2 years before COVID-19 diagnosis, but those included may have discontinued treatment before COVID-19 diagnosis. Baseline systemic therapies are those received within the 6 months before COVID-19 diagnosis; patients may have been receiving a TNFi, non-TNFi biologic, or JAK inhibitor in combination with a csDMARD. Patients in each indicated cohort are mutually exclusive. Patients with $\geq 1$ overlapping diagnosis codes for the indicated conditions during baseline are not included in the analysis. JAK inhibitors included tofacitinib, baricitinib, and upadacitinib. TNFi included adalimumab, certolizumab pegol, etanercept, golimumab, and infliximab. Non-TNFi biologics included sarilumab, ustekinumab, secukinumab, abatacept, tocilizumab, ixekizumab, and rituximab. csDMARDs included auranofin, aurothioglucose, azathioprine, chloroquine hydrochloride, chloroquine phosphate, cyclophosphamide, cyclosporine, gold sodium thiomalate, hydroxychloroquine sulfate, leflunomide, mercaptopurine, mesalamine, methotrexate, minocycline hydrochloride, $n$-acetylpenicillamine, penicillamine, primaquine, sulfasalazine, tacrolimus, and thalidomide. csDMARD: conventional synthetic disease-modifying antirheumatic drug; COVID-19: coronavirus disease 2019; JAK: Janus kinase; N: number of patients in the cohort; $n$ : number of patients in the specified category; PsA: psoriatic arthritis; RA: rheumatoid arthritis; SARS-CoV-2: severe acute respiratory syndrome coronavirus 2; TNFi: tumor necrosis factor inhibitor; UC: ulcerative colitis. 
Table 1. Baseline demographics and characteristics of patients with COVID-19 in the indicated and comparator cohorts.

\begin{tabular}{|c|c|c|c|c|}
\hline & \multicolumn{3}{|c|}{ Indicated Cohorts } & \multirow{2}{*}{$\begin{array}{c}\text { Comparator Cohort } \\
n=311,563\end{array}$} \\
\hline & $\mathrm{RA}, \mathrm{n}=2306$ & PsA, $n=421$ & $\mathrm{UC}, \mathrm{n}=811$ & \\
\hline Age, yrs, median (range) & $61(19-89)$ & $55(22-89)$ & $52(18-89)$ & $49(18-89)$ \\
\hline \multicolumn{5}{|l|}{ Race } \\
\hline White & $1634(70.9)$ & $367(87.2)$ & $651(80.3)$ & $218,293(70.1)$ \\
\hline Black or African American & $356(15.4)$ & $16(3.8)$ & $83(10.2)$ & $40,407(13.0)$ \\
\hline \multicolumn{5}{|l|}{ US region } \\
\hline Midwest & $988(42.8)$ & $167(39.7)$ & $397(49.0)$ & $156,441(50.2)$ \\
\hline Northeast & $749(32.5)$ & $199(47.3)$ & $265(32.7)$ & $80,041(25.7)$ \\
\hline South & $346(15.0)$ & $33(7.8)$ & $77(9.5)$ & $47,212(15.2)$ \\
\hline West & $174(7.6)$ & $15(3.6)$ & $56(6.9)$ & $17,521(5.6)$ \\
\hline Medicare & $1054(45.7)$ & $83(19.7)$ & $222(27.4)$ & $62,068(19.9)$ \\
\hline Other payor type & $396(17.2)$ & $61(14.5)$ & $112(13.8)$ & $45,209(14.5)$ \\
\hline Uninsured & $176(7.6)$ & $36(8.6)$ & $59(7.3)$ & $25,818(8.3)$ \\
\hline Unknown & $1526(66.2)$ & $294(69.8)$ & $552(68.1)$ & $142,854(45.9)$ \\
\hline \multicolumn{5}{|l|}{ Baseline systemic treatment ${ }^{c}$} \\
\hline Tofacitinib & $69(3.0)$ & $13(3.1)$ & $11(1.4)$ & $0(0.0)$ \\
\hline JAK inhibitors $^{\mathrm{d}}$ & $96(4.2)$ & $13(3.1)$ & $11(1.4)$ & $0(0.0)$ \\
\hline $\mathrm{TNFi}^{\mathrm{e}}$ & $228(9.9)$ & $103(24.5)$ & $76(9.4)$ & $0(0.0)$ \\
\hline Non-TNFi biologic ${ }^{f}$ & $278(12.1)$ & $75(17.8)$ & $145(17.9)$ & $0(0.0)$ \\
\hline csDMARD $^{\circ}$ & $838(36.3)$ & $93(22.1)$ & $254(31.3)$ & $0(0.0)$ \\
\hline Corticosteroid use $\mathrm{e}^{\mathrm{h}}$ & $748(32.4)$ & $85(20.2)$ & $198(24.4)$ & $19,651(6.3)$ \\
\hline \multicolumn{5}{|l|}{ Comorbidities and medical history } \\
\hline Serious infections (hospitalized) & $201(8.7)$ & $13(3.1)$ & $86(10.6)$ & $6952(2.2)$ \\
\hline Cancer & $179(7.8)$ & $19(4.5)$ & $63(7.8)$ & $8907(2.9)$ \\
\hline Other immune deficiencies & $120(5.2)$ & $13(3.1)$ & $31(3.8)$ & $864(0.3)$ \\
\hline COPD & $288(12.5)$ & $20(4.8)$ & $50(6.2)$ & $9625(3.1)$ \\
\hline Liver disease & $117(5.1)$ & $22(5.2)$ & $47(5.8)$ & $5391(1.7)$ \\
\hline ILD & $86(3.7)$ & $4(1.0)$ & $16(2.0)$ & $956(0.3)$ \\
\hline HIV and/or AIDS ${ }^{j}$ & $<10(<1)$ & $0(0.0)$ & $<10(<1)$ & $841(0.3)$ \\
\hline
\end{tabular}

Data are $\mathrm{n}(\%)$ unless otherwise indeicated. ${ }^{\mathrm{a}}$ Baseline was defined as within 6 months before COVID-19 diagnosis date. ${ }^{\mathrm{b}}$ May not be mutually exclusive for each insurance type. ${ }^{c}$ Patients may have been receiving TNFi, non-TNFi biologic, or JAK inhibitor in combination with a csDMARD. ${ }^{\mathrm{d}}$ JAK inhibitors included tofacitinib, baricitinib, and upadacitinib. ${ }^{\mathrm{e}}$ TNFi included adalimumab, certolizumab pegol, etanercept, golimumab, and infliximab. ${ }^{\mathrm{f}}$ Non-TNFi biologics included sarilumab, ustekinumab, secukinumab, abatacept, tocilizumab, ixekizumab, and rituximab. ${ }^{\mathrm{g}} \mathrm{csDMARD}$ included auranofin, aurothioglucose, azathioprine, chloroquine hydrochloride, chloroquine phosphate, cyclophosphamide, cyclosporine, gold sodium thiomalate, hydroxychloroquine sulphate, leflunomide, mercaptopurine, mesalamine, methotrexate, minocycline hydrochloride, $\mathrm{n}$-acetylpenicillamine, penicillamine, primaquine, sulfasalazine, tacrolimus, and

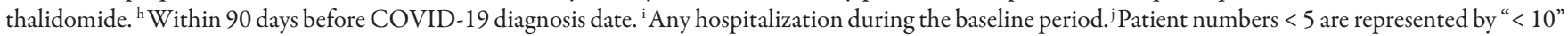
in tables to protect patient anonymity. AIDS: acquired immunodeficiency syndrome; COPD: chronic obstructive pulmonary disease; csDMARD: conventional synthetic disease-modifying antirheumatic drug; HIV: human immunodeficiency virus; ILD: interstitial lung disease; JAK: Janus kinase; PsA: psoriatic arthritis; RA: rheumatoid arthritis; TNFi: tumor necrosis factor inhibitor; UC: ulcerative colitis; VTE: venous thromboembolism. 

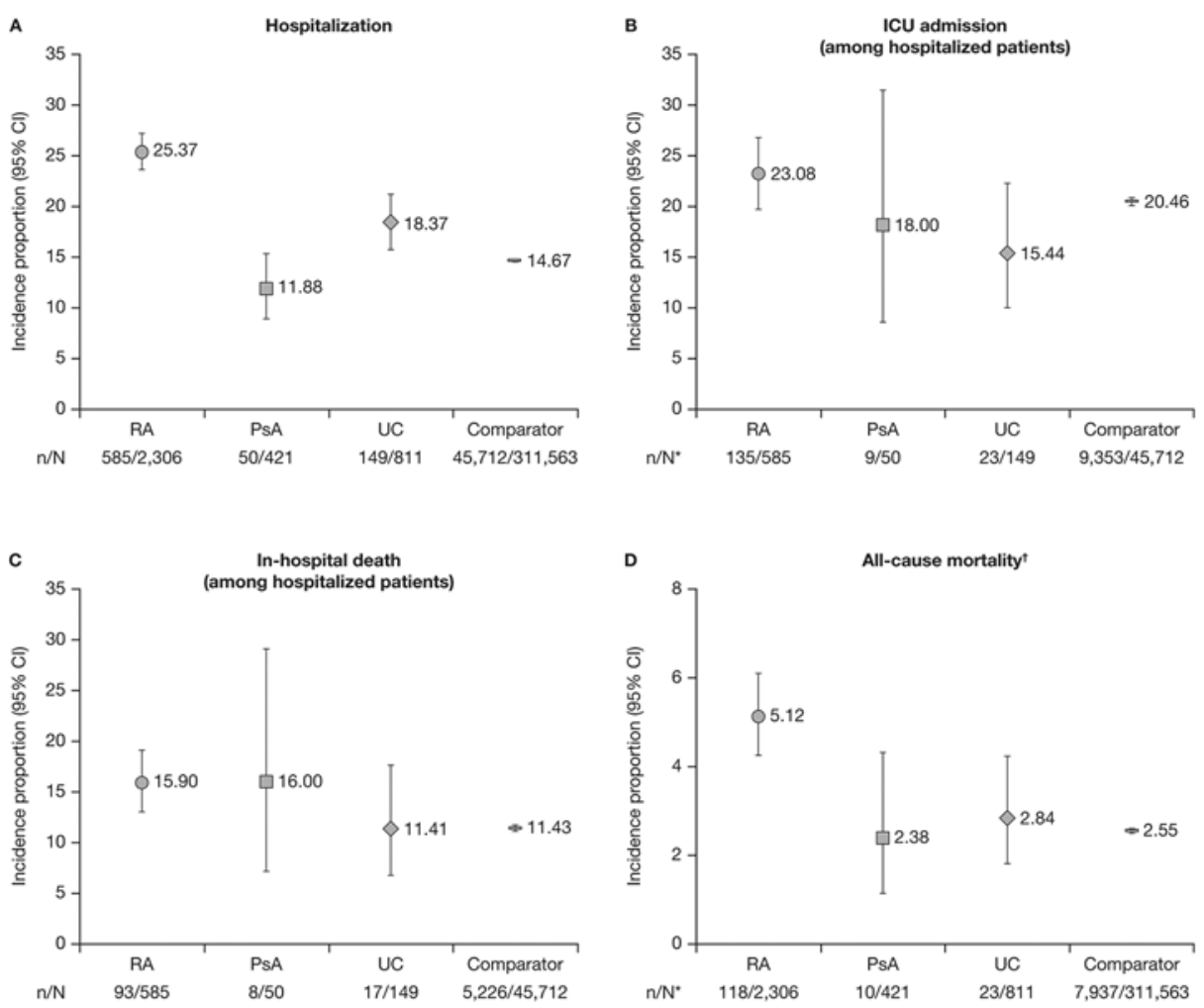

Figure 2. Crude incidence of (A) hospitalization, (B) ICU admission, (C) in-hospital death, and (D) all-cause mortality in the indicated and comparator cohorts. "Denominator is the number of patients hospitalized. ${ }^{\dagger}$ Within 90 days before COVID-19 diagnosis. COVID-19: coronavirus disease 2019; ICU: intensive care unit; $N$ : number of patients in the cohort; $\mathrm{n}$ : number of patients in the specified category; PsA: psoriatic arthritis; RA: rheumatoid arthritis; UC: ulcerative colitis.

admission (conditional on hospitalization) for each cohort are shown in Figures 2A,B.

The proportions of COVID-19 patients with RA who were hospitalized and receiving baseline systemic therapy were: $104 / 278$ patients receiving non-TNFi at baseline $(37.4 \%, 95 \%$ CI 31.7-43.4), 190/838 patients receiving csDMARDs (22.7\%, 95\% CI 19.9-25.7), 12/69 patients receiving tofacitinib (17.4\%, 95\% CI 9.3-28.4), and 29/228 patients receiving TNFi (12.7\%, 95\% CI 8.7-17.8; Supplementary Figure 2, available with the online version of this article). Among hospitalized patients with RA receiving baseline systemic therapy, ICU admission was required in $5 / 12$ patients receiving tofacitinib $(41.7 \%, 95 \% \mathrm{CI}$ 15.2-72.3), 49/190 receiving csDMARDs $(25.8 \%$, 95\% CI 19.7-32.6), 21/104 receiving non-TNFi biologics (20.2\%, 95\% CI 13.0-29.2]), and 5/29 receiving TNFi (17.2\% [5.9-35.8]; Supplementary Figure 2).

A similar trend was noted in both the PsA and UC cohorts (Supplementary Figure 2, available with the online version of this article).

Length of hospital stay. The length of hospital stay in the indicated cohort varied by immune-mediated inflammatory condition, with $67-76 \%$ of patients across the indicated and comparator cohorts having a hospital stay between 1 and 10 days (Supplementary Figure 3 , available with the online version of this article). The mean length of hospital stay among patients with COVID-19 was 9.7, 7.7, and 9.0 days in the RA, PsA, and UC cohorts, respectively.
Crude incidence of in-hospital deaths and all-cause mortality. The crude incidence of in-hospital deaths and all-cause mortality in patients with COVID-19 for each cohort are shown in Figures 2C,D. The proportion of deaths varied by immune-mediated inflammatory condition and baseline systemic therapy (Supplementary Figure 2, available with the online version of this article).

Crude incidence of COVID-19-related clinical manifestations and outcomes of interest. The proportion of patients diagnosed with other clinical manifestations or outcomes of interest in the indicated cohorts varied by immune-mediated inflammatory conditions (Figure 3) and baseline therapy (Supplementary Figures 4-6, available with the online version of this article).

When stratified by baseline therapy, the number of patients with COVID-19-related clinical manifestations or outcomes of interest was small (Supplementary Figures 4-6, available with the online version of this article).

Risk of COVID-19-related outcomes. Adjusting for demographics, COVID-19 patients with RA had an increased risk of hospitalization and in-hospital death compared with the comparator cohort (Figure 4A). This risk was somewhat attenuated when adjusted for demographics plus comorbidities. With adjustment for demographics and demographics plus comorbidities, patients with RA also had an increased risk of ICU admission, pneumonia, thrombotic event, ARDS, heart failure, sepsis or septic shock, and mechanical ventilation or ECMO (Figure $4 \mathrm{~A}$ ). When analyses 

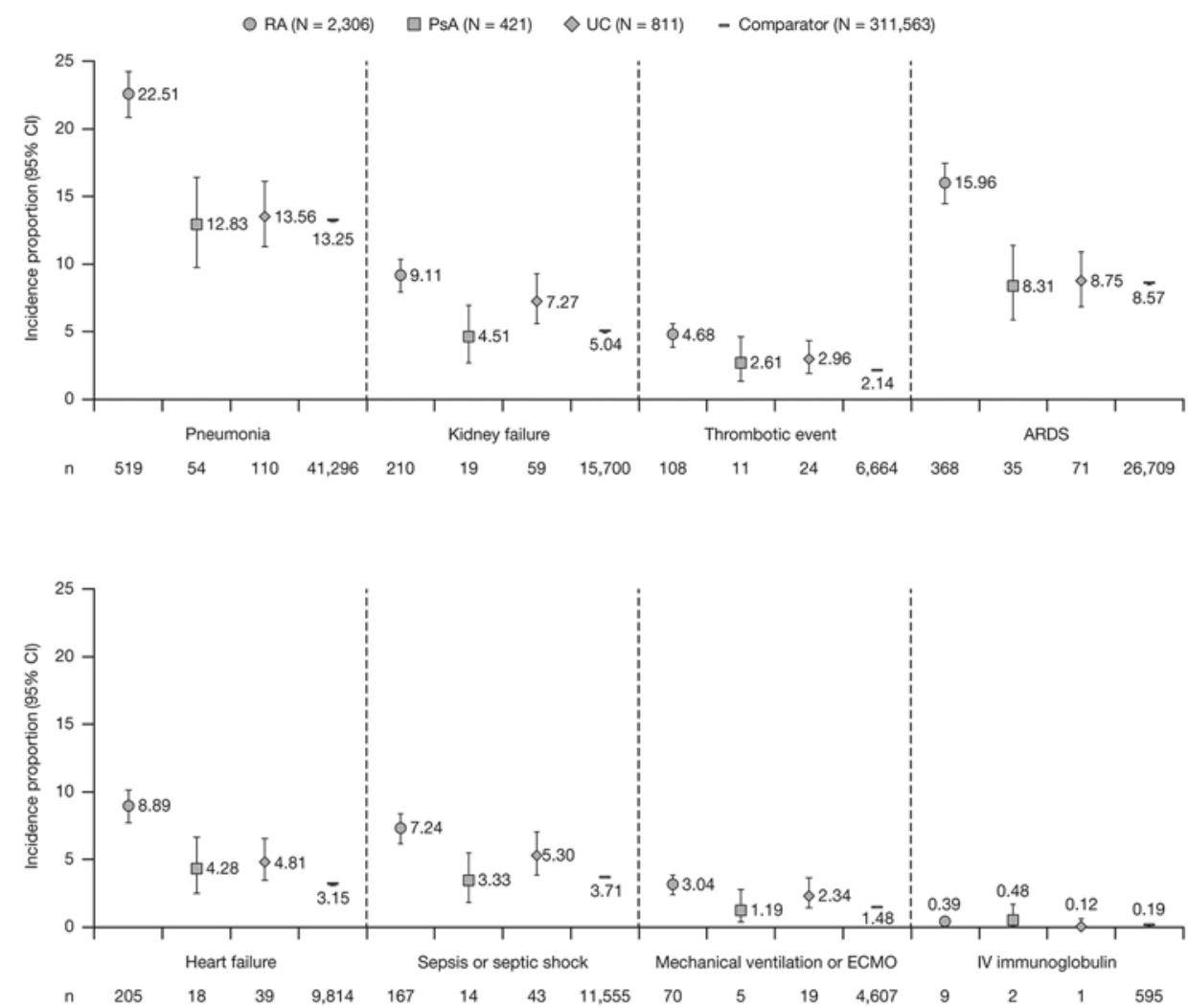

Figure 3. Crude incidence of COVID-19-related clinical manifestations and outcomes of interest in the indicated and comparator cohorts. Intravenous Ig was used as an indicator of severe COVID-19. ARDS: acute respiratory distress syndrome; COVID-19: coronavirus disease 2019; ECMO: extracorporeal membrane oxygenation; $\mathrm{N}$ : number of patients in the cohort; $n$ : number of patients in the specified category; PsA: psoriatic arthritis; RA: rheumatoid arthritis; UC: ulcerative colitis.

were restricted to hospitalized patients, the risk of ICU admission, mechanical ventilation or ECMO, and in-hospital death was attenuated. The risk of ICU admission and mechanical ventilation or ECMO was no longer significant when conditional on hospitalization and adjusted for demographics plus comorbidities.

Adjusting for demographics, patients with UC had an increased risk of hospitalization; however, after adjustment for demographics plus comorbidities, the risk was no longer statistically significant (Figure 4B). Compared with the comparator cohort, there was no statistically significant increased risk of hospitalization among patients with PsA (Figure 4C).

When analyzed by baseline systemic immunomodulatory therapy, after adjustment for demographics plus comorbidities, the risk of hospitalization was lower in patients with RA receiving TNFi relative to patients receiving non-TNFi biologics (Figure 5A). Patients with RA receiving csDMARDs had an increased risk of hospitalization relative to the comparator cohort. The adjusted risk of hospitalization in patients with RA was similar between patients receiving JAK inhibitors or tofacitinib and TNFi, and between patients receiving tofacitinib and the comparator cohort (Figure 5A). Similar findings were observed when patients from the 3 indicated cohorts were combined, although patients receiving JAK inhibitors had an increased risk of hospitalization relative to patients receiving TNFi (Figure 5B).

\section{DISCUSSION}

We present data from patients with COVID-19 between February 1 and December 9, 2020, utilizing the US Optum deidentified COVID-19 EHR dataset, to establish whether patients with COVID-19 and inflammatory conditions who were receiving tofacitinib or other immunomodulatory therapies had more severe outcomes compared with a general population cohort with COVID-19. To our knowledge, this is the largest study using real-world data to evaluate the outcomes of COVID-19 in patients with conditions treated with systemic immunomodulatory therapies, relative to the general population.

Baseline comorbid conditions were more frequent among the indicated cohort patients than in the comparator cohort. Higher incidences of hospitalizations (25.4\%) and ICU admissions (23.1\%) likely due to COVID-19 were noted among patients with RA, compared with the other cohorts. COVID-19 patients with RA demonstrated higher risk of outcomes associated with more severe COVID-19 disease, including hospitalization, ICU admission, in-hospital death, mechanical ventilation or ECMO, and other clinical manifestations, relative to the comparator cohort after adjustment for demographics and demographics plus comorbidities. There was no evidence of increased risk of hospitalization or clinical manifestations related to severe COVID-19 in patients with PsA. 


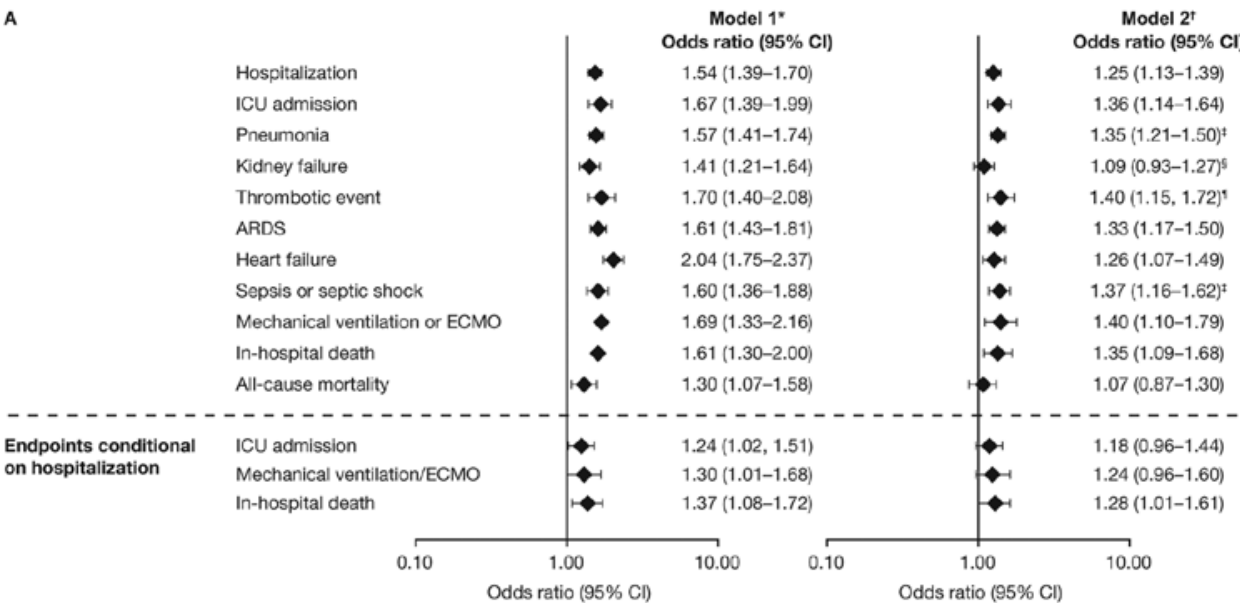

B

Model $1 *$

Odds ratio $(95 \% \mathrm{CI})$

$1.31(1.09-1.58)$

$0.94(0.62-1.44)$

$1.01(0.82-1.25)$

$1.43(1.08-1.90)$

$1.28(0.85-1.93)$

$0.98(0.76-1.27)$

$1.42(1.01-1.98)$

$1.38(1.00-1.89)$

$1.50(0.94-2.37)$

$1.11(0.68-1.82)$

$0.98(0.64-1.50)$

- - - - - - -

$0.77(0.49-1.21)$

$1.25(0.77-2.03)$

$0.95(0.56-1.60)$
10.00

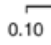

0.1

Model 1*

c

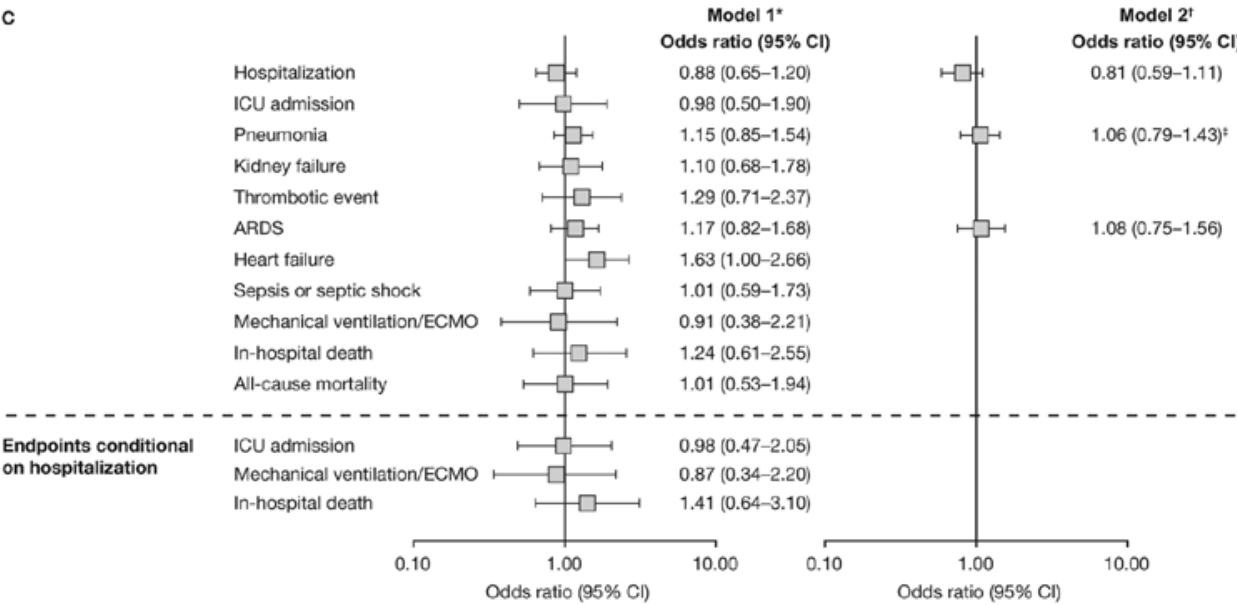

Figure 4. Adjusted ORs of each endpoint vs the comparator cohort in (A) the RA cohort, (B) the UC cohort, and (C) the PsA cohort. " Covariates included in logistic regression models were age, gender, race, and region. ${ }^{\dagger}$ Covariates included in logistic regression models were age, sex, race, region, lung disease (interstitial lung disease, asthma, and chronic obstructive pulmonary disease), venous thromboembolism, hypertension, coronary artery disease, serious infections (hospitalized), cancer, diabetes, CKD or dialysis, and liver disease (except for UC, which did not include serious infections [hospitalized]). ORs using this model are only displayed for endpoints with > 30 events for each endpoint or outcome in the RA, PsA, and UC cohorts. The risk of ICU admission, mechanical ventilation or ECMO, and in-hospital death conditional on hospitalization in UC and PsA cohorts was estimated with adjustment for demographics only. ${ }^{\ddagger}$ Serious infections (hospitalized) were not included as a covariate. ${ }^{\S} \mathrm{CKD} / \mathrm{dialysis}$ was not included as a covariate. 'Venous thromboembolism was not included as a covariate. ARDS: acute respiratory distress syndrome; CKD: chronic kidney disease; COVID-19: coronavirus disease 2019; ECMO: extracorporeal membrane oxygenation; ICU: intensive care unit; PsA: psoriatic arthritis; RA: rheumatoid arthritis; UC: ulcerative colitis. 


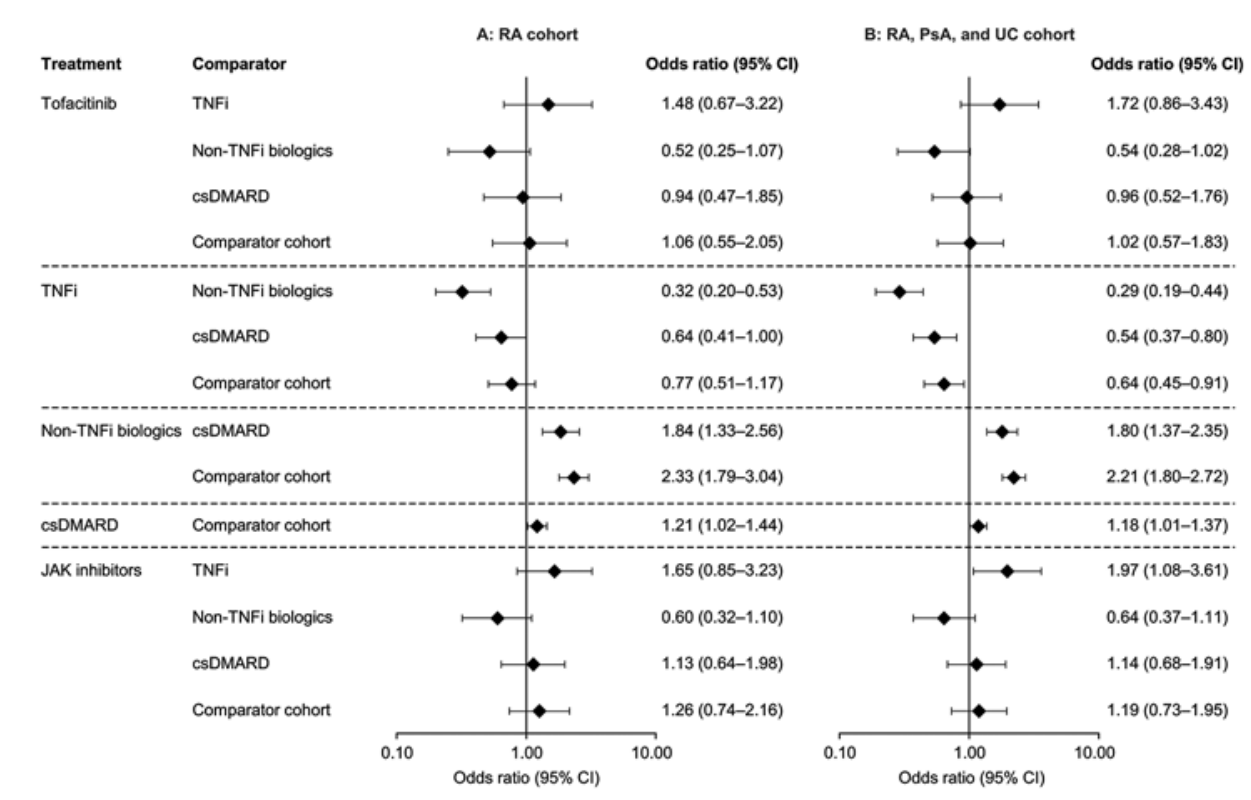

Figure 5. Adjusted ORs of hospitalization according to baseline systemic immunomodulatory therapy in (A) the RA cohort and (B) the indicated cohorts combined. Covariates included in logistic regression models were age, sex, race, region, hypertension, diabetes, and chronic kidney disease or dialysis. The number of hospitalizations in each treatment group is $\geq 10$. csDMARD: conventional synthetic disease-modifying antirheumatic drug; JAK: Janus kinase; PsA: psoriatic arthritis; RA: rheumatoid arthritis; TNFi: tumor necrosis factor inhibitor; UC: ulcerative colitis.

The COVID-19 Global Rheumatology Alliance (GRA) registry reported that 49\% (1739/3549) of patients with COVID-19 and RA required hospitalization, ${ }^{6}$ and identified older age ( $\geq 65 \mathrm{yrs})$ and the presence of comorbidities such as hypertension, cardiovascular disease, and diabetes, as risk factors. ${ }^{7}$ The hospitalization rate due to COVID-19 in patients with RA in our US study was lower than that reported in the GRA registry (25.4\% vs $49.0 \%$ ), although differences in data collection may account for this disparity. ${ }^{7}$ In our present study, the incidence of hospitalization in COVID-19 patients with PsA was lower than that in COVID-19 patients with RA, and similar to the comparator cohort. Similarly, Haberman et al found that patients with COVID-19 and RA had a higher hospitalization rate than patients with COVID-19 and spondyloarthritis, ${ }^{8}$ whereas others have reported similar rates of hospitalization due to COVID-19 in patients with and without rheumatic diseases. ${ }^{9} 10$ Further, patients with rheumatic diseases have been identified to be at increased risk of requiring ICU admission or mechanical ventilation for COVID-19 compared with patients without rheumatic diseases. ${ }^{9}$ It is unclear why patients with RA are at increased risk of hospitalization or ICU admission, although RA has also previously been identified as a risk factor for death related to COVID-19. ${ }^{11}$ In our study, the exploratory analyses used 2 different models, the first with adjustment for demographics and the second with adjustment for demographics plus comorbidities. The comorbidity covariates included in the second model were selected by considering their clinical relevance, the current literature, and statistical relevance including the events per variable rule. In general, the addition of comorbidities resulted in a $<20 \%$ reduction in the ORs, suggesting that the additional comorbidity covariates may not be large confounders for the majority of endpoints in this analysis. The ACR suggests that the adjustment for comorbidities, which are shared risk factors for autoimmune and inflammatory rheumatic diseases, may be inappropriate and may lead to the underestimation of the risk of COVID-19 in these patients. ${ }^{4}$ In our present study, ILD, which may be a direct manifestation associated with some autoimmune and inflammatory rheumatic diseases, was included as a covariate in the model adjusted for demographics plus comorbidities; however, exploratory analyses in which ILD was excluded as a covariate yielded similar results (data not shown).

Hospitalization rates for COVID-19 in patients with inflammatory bowel disease (IBD) have previously been reported as $20-67 \%^{12,13}$; a lower hospitalization rate was reported in our study (18.4\%) in patients with UC. Consistent with findings reported here, a previous study has also reported the risk of severe outcomes among COVID-19 patients with IBD to be comparable to the general population. ${ }^{14}$

The GRA registry showed that, in patients with rheumatic diseases, TNFi (alone or in combination with csDMARDs) treatment was associated with reduced odds of hospitalization due to COVID-19, ${ }^{7}$ and compared with methotrexate therapy, treatment with TNFi was not associated with COVID-19-related death. However, treatment with rituximab (RTX) was associated with higher odds of COVID-19-related death. ${ }^{6}$ In patients with RA, treatment with RTX or JAK inhibitors led to worse COVID-19 severity outcomes compared with TNFi. ${ }^{15}$ In the Surveillance Epidemiology of Coronavirus Under Research Exclusion (SECURE)-IBD population, TNFi use was not associated with severe COVID-19, but was inversely associated with the composite outcome of death or hospital 
admission. ${ }^{13}$ In our study, we noted a numerically lower incidence of more severe COVID-19 and death in patients with RA and UC receiving TNFi, compared with non-TNFi biologics. We observed that the incidence of ICU admission was numerically higher in hospitalized patients with RA receiving tofacitinib compared with other therapies, although the number of hospitalized patients with RA across treatment groups was low. In adjusted analyses, the risk of hospitalization in patients with RA was higher among patients receiving non-TNFi biologics compared with patients receiving $\mathrm{TNFi}$; this was also true when patients from the 3 indicated cohorts were combined. The risk of hospitalization was similar among patients with RA receiving JAK inhibitors or tofacitinib and patients receiving TNFi, and for patients receiving JAK inhibitors or tofacitinib and the comparator cohort, although the CIs were wide. Similar findings have been reported from the SECURE-IBD registry in patients with IBD receiving tofacitinib vs other IBD therapies. ${ }^{16}$

COVID-19 vaccines have been allocated to priority groups based on epidemiologic data and consideration of those at higher risk of severe disease and/or death. The findings presented here, including that patients with RA are more likely to be hospitalized, strengthen the need for these patients to be prioritized in initial COVID-19 vaccine program planning.

Limitations of our data include their derivation from EHRs and the identification of immune-mediated immunomodulatory conditions using ICD-10-CM diagnosis codes, and the short duration of the baseline period of the study. The small sample size, especially when stratified by baseline systemic therapy and size differences of the 4 cohorts, also limits the interpretation of the data. The validity of diagnosis codes for SARS-CoV-2 infection is established in the hospitalized patient, but they lack sensitivity for outpatient infection. ${ }^{17}$ Active SARS-CoV-2 infection was diagnosed with a positive test or positive antibody test, and diagnostic misclassification of active infection due to false positive tests is a potential source of bias. The spectrum of clinical manifestations of SARS-CoV-2 infection is unknown, and many infected individuals may not seek medical care. Exposure to, and testing for, SARS-CoV-2 infection may be associated with the underlying conditions of the indicated patients and has the potential to introduce bias. Higher-risk patients with autoimmune and inflammatory conditions may have been more likely to be tested, especially early in the course of the pandemic. Thus, our results may not be generalizable to less severe disease. Further, the disease activity of patients included in the indicated cohorts of this study was unknown. Since some comparisons were limited by small sample size and/or few events, a full adjustment of demographics and comorbidities may not be appropriate. Additionally, BMI (a known predictor for more severe COVID-19 ${ }^{18}$ ) was not included as a covariate in the exploratory analyses reported here, due to data unavailability.

The data reported here add evidence to the current literature that patients with RA, but not PsA or UC, are at higher risk for more severe or critical COVID-19. Patients treated with systemic therapies did not appear to be at increased risk of severe COVID-19 compared to the comparator cohort, with the possible exception of non-TNFi biologics. However, more data are needed to further understand the risk and outcome of COVID-19 in these patient populations. Collectively, these data from patients with immune-mediated inflammatory conditions are important for informing medical management strategies, as well as COVID-19 vaccine priority decisions.

\section{ACKNOWLEDGMENT}

The authors thank Rongjun Shen, Kathy Liu, and Ling Li for their contribution to the analyses and quality control for this study, and Jasper Deuring, Dayo Jagun, and Joseph Cappelleri for their clinical and statistical input. This study was sponsored by Pfizer Inc. Medical writing support, under the guidance of the authors, was provided by Helen Findlow, PhD, CMC Connect, McCann Health Medical Communications, and was funded by Pfizer Inc, New York, NY, USA in accordance with Good Publication Practice (GPP3) guidelines (Ann Intern Med 2015;163:461-4).

\section{DATA SHARING POLICY}

Upon request, and subject to review, Pfizer will provide the data that support the findings of this study. Subject to certain criteria, conditions, and exceptions, Pfizer may also provide access to the related individual anonymized participant data. See https://www.pfizer.com/science/clinical-trials/ trial-data-and-results for more information.

\section{ONLINE SUPPLEMENT}

Supplementary material accompanies the online version of this article.

\section{REFERENCES}

1. Listing J, Gerhold K, Zink A. The risk of infections associated with rheumatoid arthritis, with its comorbidity and treatment. Rheumatology 2013;52:53-61.

2. Rahier JF, Magro F, Abreu C, et al; European Crohn's and Colitis Organisation (ECCO). Second European evidence-based consensus on the prevention, diagnosis and management of opportunistic infections in inflammatory bowel disease. J Crohns Colitis 2014;8:443-68.

3. Minozzi S, Bonovas S, Lytras T, et al. Risk of infections using anti-TNF agents in rheumatoid arthritis, psoriatic arthritis, and ankylosing spondylitis: a systematic review and meta-analysis. Expert Opin Drug Saf 2016;15:11-34.

4. Curtis JR, Johnson SR, Anthony DD, et al. American College of Rheumatology guidance for COVID-19 vaccination in patients with rheumatic and musculoskeletal diseases: version 1. Arthritis Rheumatol 2021;73:1093-107.

5. Wallace PJ, Shah ND, Dennen T, Bleicher PA, Crown WH. Optum Labs: building a novel node in the learning health care system. Health Aff 2014;33:1187-94.

6. Strangfeld A, Schäfer M, Gianfrancesco MA, et al; COVID-19 Global Rheumatology Alliance. Factors associated with COVID-19-related death in people with rheumatic diseases: results from the COVID-19 Global Rheumatology Alliance physician-reported registry. Ann Rheum Dis 2021;80:930-42.

7. Gianfrancesco M, Hyrich KL, Al-Adely S, et al; COVID-19 Global Rheumatology Alliance. Characteristics associated with hospitalisation for COVID-19 in people with rheumatic disease: data from the COVID-19 Global Rheumatology Alliance physician-reported registry. Ann Rheum Dis 2020;79:859-66.

8. Haberman RH, Castillo R, Chen A, et al; NYU WARCOV Investigators. COVID-19 in patients with inflammatory arthritis: a prospective study on the effects of comorbidities and disease-modifying antirheumatic drugs on clinical outcomes. Arthritis Rheumatol 2020;72:1981-9. 
9. D'Silva KM, Serling-Boyd N, Wallwork R, et al. Clinical characteristics and outcomes of patients with coronavirus disease 2019 (COVID-19) and rheumatic disease: a comparative cohort study from a US 'hot spot'. Ann Rheum Dis 2020;79:1156-62.

10. Pablos JL, Galindo M, Carmona L, et al; RIER Investigators Group. Clinical outcomes of hospitalised patients with COVID-19 and chronic inflammatory and autoimmune rheumatic diseases: a multicentric matched cohort study. Ann Rheum Dis 2020; 79:1544-9.

11. Topless RK, Phipps-Green A, Leask M, et al. Gout, rheumatoid arthritis, and the risk of death related to coronavirus disease 2019: an analysis of the UK Biobank. ACR Open Rheumatol 2021; 3:333-40.

12. Aziz M, Fatima R, Haghbin H, Lee-Smith W, Nawras A. The incidence and outcomes of COVID-19 in IBD patients: a rapid review and meta-analysis. Inflamm Bowel Dis 2020;26:e132-3.

13. Brenner EJ, Ungaro RC, Gearry RB, et al. Corticosteroids, but not TNF antagonists, are associated with adverse COVID-19 outcomes in patients with inflammatory bowel diseases: results from an international registry. Gastroenterology 2020;159:481-91.

14. Singh S, Khan A, Chowdhry M, Bilal M, Kochhar GS, Clarke K. Risk of severe coronavirus disease 2019 in patients with inflammatory bowel disease in the United States: a multicenter research network study. Gastroenterology 2020;159:1575-8.e4.

15. Sparks JA, Wallace ZS, Seet AM, et al; COVID-19 Global Rheumatology Alliance. Associations of baseline use of biologic or targeted synthetic DMARDs with COVID-19 severity in rheumatoid arthritis: results from the COVID-19 Global Rheumatology Alliance physician registry. Ann Rheum Dis 2021;80:1137-46.

16. Agrawal M, Brenner EJ, Zhang X, et al. Characteristics and outcomes of IBD patients with COVID-19 on tofacitinib therapy in the SECURE-IBD registry. Inflamm Bowel Dis 2021;27:585-9.

17. Kluberg S. Validation of claims-based algorithms to identify hospitalized COVID-19 events within the FDA Sentinel system. [Internet. Accessed November 16, 2021]. Available from: https:// www.sentinelinitiative.org/sites/default/files/communications/ publications-presentations/Validation_of_claims_based_algorithms. pdf

18. Kim TS, Roslin M, Wang JJ, Kane J, Hirsch JS, Kim EJ; Northwell Health COVID-19 Research Consortium. BMI as a risk factor for clinical outcomes in patients hospitalized with COVID-19 in New York. Obesity 2021;29:279-84. 